\title{
Study of $\tau$ Decays to Four-Hadron Final States with Kaons
}

\section{K.K. Gan*}

The Ohio State University, USA

E-mail: gan@mps.ohio-state.edu

The rare $\tau$ lepton decays to four explicitly identified hadrons have been studied with the CLEO detector at the Cornell Electron Storage Ring (CESR) using $(7.56 \pm 0.15) \mathrm{fb}^{-1}$ of data collected near $\sqrt{s}=10.58 \mathrm{GeV}$. The first statistically significant measurements of $\mathcal{B}\left(\tau^{-} \rightarrow\right.$ $K^{-} \pi^{+} \pi^{-} \pi^{0} \nu_{\tau}$, excluding $\left.K^{0}\right)=(7.4 \pm 0.8 \pm 1.1) \times 10^{-4}$ and $\mathcal{B}\left(\tau^{-} \rightarrow K^{-} K^{+} \pi^{-} \pi^{0} \nu_{\tau}\right)=$ $(5.5 \pm 1.4 \pm 1.2) \times 10^{-5}$ are presented, including the first observation of the decay $\tau^{-} \rightarrow K^{-} \omega \nu_{\tau}$ with branching fraction, $(4.1 \pm 0.6 \pm 0.7) \times 10^{-4}$. We also publish the first upper limit for $\mathcal{B}\left(\tau^{-} \rightarrow K^{-} K^{+} K^{-} \pi^{0} \nu_{\tau}\right)<4.8(6.1) \times 10^{-6}$ at $90 \%$ (95\%) confidence level (C.L.). We also measure for the first time the invariant mass distributions of the decays.

International Europhysics Conference on High Energy Physics

July 21st - 27th 2005

Lisboa, Portugal

${ }^{*}$ Representing the CLEO III Collaboration. This work was supported by the National Science Foundation and the U.S. Department of Energy. 
The suppressed decays of the $\tau$ lepton to final states with kaons provide a powerful probe of the strange sector of the weak charged current. Measurement of the branching fractions and invariant mass distributions of various strange final states are needed to extract the Standard Model parameters, the strange quark mass $m_{s}$ and the Cabibbo-Kobayashi-Maskawa element $V_{u s}$, from $\tau$ lepton decays with precision competitive with both $K_{e 3}$ decay and lattice determinations [1]. In this paper, we present the first statistically significant measurements of the branching fractions for the decays $\tau^{-} \rightarrow K^{-} \pi^{+} \pi^{-} \pi^{0} \nu_{\tau}$ (ex. $K^{0}$ ) [2] and $K^{-} K^{+} \pi^{-} \pi^{0} \nu_{\tau}$, as well as the first published upper limit for the decay $\tau^{-} \rightarrow K^{-} K^{+} K^{-} \pi^{0} \nu_{\tau}$. We also investigate the substructure of the decays $\tau^{-} \rightarrow K^{-} \pi^{+} \pi^{-} \pi^{0} \nu_{\tau}$ and $K^{-} K^{+} \pi^{-} \pi^{0} \nu_{\tau}$, yielding the first observation and measurement of $\tau^{-} \rightarrow K^{-} \omega \nu_{\tau}$. We also present the first measurements of the invariant mass distributions of the decays. The branching fraction for the decay $\tau^{-} \rightarrow K^{-} \omega \nu_{\tau}$ is also compared to the prediction of $\mathrm{Li}[3]$ and find significant disagreement.

The data used in this analysis were collected with the CLEO III detector [4] at CESR near the center-of-mass energy $10.58 \mathrm{GeV}$. The sample corresponds to an integrated luminosity of $(7.56 \pm$ $0.15) \mathrm{fb}^{-1}$ containing $(6.90 \pm 0.14) \times 10^{6} \tau$-pair events produced in $e^{+} e^{-}$collisions. CLEO III is a general purpose detector with a Ring Imaging Cherenkov (RICH) detector [5] that is critical for the $\pi / K$ separation used in this analysis. We select events with four charged tracks in the 1-vs-3 topology. There must be at least one $\pi^{0}$ candidate in the 3-prong hemisphere. We do not explicitly veto events based on the photon multiplicity in order to reduce the dependence on Monte Carlo simulation of fake photons. Each charged particle in the 3-prong hemisphere must be identified as either a $\pi$ or $K$ candidate. For candidate $\tau^{-} \rightarrow K^{-} \omega \nu_{\tau}$ decays, we only identify the bachelor track and assume the tracks used to construct $\omega$ candidates are $\pi$ 's. For $\tau^{-} \rightarrow K^{-} \pi^{+} \pi^{-} \pi^{0} \nu_{\tau}$ (ex. $\omega$ ) decays, we reject events with $M\left(\pi^{+} \pi^{-} \pi^{0}\right)$ within $30 \mathrm{MeV} / c^{2}$ of the nominal $\omega$ mass.

Efficiencies and $\tau$-migration backgrounds are estimated using Monte Carlo events generated from the KORALB-TAUOLA program [6] with the detector response simulated by the GEANT program [7]. We model the decay $\tau^{-} \rightarrow K^{-} \pi^{+} \pi^{-} \pi^{0} \nu_{\tau}$ (ex. $\omega$ ) with the $K^{-} a_{1}^{0}$ intermediate state and the decays $\tau^{-} \rightarrow K^{-} K^{+} \pi^{-} \pi^{0} \nu_{\tau}$ and $K^{-} K^{+} K^{-} \pi^{0} \nu_{\tau}$ via phase space. The decay $\tau^{-} \rightarrow K^{-} \omega \nu_{\tau}$ is modelled with an equal mixture of $K_{1}(1270)$ and $K_{1}(1400)$ resonances. Hadronic backgrounds are calculated empirically using a sample of high-mass tagged events assuming the two jets fragment independently. Efficiencies and fake rates in the Monte Carlo simulation are calibrated using kinematically selected $D^{*+} \rightarrow D^{0} \pi^{+}$decays with $D^{0} \rightarrow K^{-} \pi^{+}$in the data. The signal, background, and detection efficiencies are summarized in Table 1. The largest sources of $\tau$-migration backgrounds are from channels with a charged pion misidentified as a kaon.

We have investigated the hadronic mass spectra to search for substructure. Figure 1(a, c, e) shows the $h^{-} h^{+} h^{-} \pi^{0}$ invariant mass spectra for $\tau^{-} \rightarrow K^{-} \pi^{+} \pi^{-} \pi^{0} \nu_{\tau}$ (ex. $\omega$ ), $K^{-} \omega \nu_{\tau}$, and $K^{-} K^{+} \pi^{-} \pi^{0} \nu_{\tau}$ events. The background- and efficiency-corrected spectra are shown in Fig. 1(b, d, f). There is no evidence for $K_{1}, \rho^{0}$, or $K^{*}$ in the decay $\tau^{-} \rightarrow K^{-} \pi^{+} \pi^{-} \pi^{0} \nu_{\tau}$ (ex. $\omega$ ). However, there is an indication of $\rho^{+}$consistent with the $K^{-} a_{1}^{0}$ model. The distribution of $M\left(\pi^{+} \pi^{-} \pi^{0}\right)$ in Fig. 2 clearly shows an $\omega$ signal, as well as a small $\eta$ meson peak consistent with expectations for the decay $\tau^{-} \rightarrow K^{-} \eta \nu_{\tau}[8,9]$. This is the first observation of the decay $\tau^{-} \rightarrow K^{-} \omega \nu_{\tau}$. The Monte Carlo model with an equal mixture of $K_{1}(1270)$ and $K_{1}(1400)$ resonances describe the $K^{-} \omega$ mass distribution well. There is no indication of $K^{*}$ or $\rho$ resonances in the decay $\tau^{-} \rightarrow K^{-} K^{+} \pi^{-} \pi^{0} \nu_{\tau}$.

In summary. we present the first statistically significant measurements of the branching frac- 


\begin{tabular}{lccccc}
\hline \hline Channel & yield & $\tau$ mig. & $q \bar{q}$ bg & $\epsilon(\%)$ & $\mathcal{B}\left(\times 10^{-4}\right)$ \\
\hline$K^{-} \pi^{+} \pi^{-} \pi^{0}$ & $833 \pm 36$ & $434 \pm 14$ & $153 \pm 25$ & $5.68 \pm 0.17$ & $3.7 \pm 0.5 \pm 0.8$ \\
$K^{-} \omega$ & $500 \pm 35$ & $194 \pm 12$ & $64 \pm 20$ & $5.61 \pm 0.09$ & $4.1 \pm 0.6 \pm 0.7$ \\
$K^{-} K^{+} \pi^{-} \pi^{0}$ & $48 \pm 9$ & $1 \pm 1$ & $9 \pm 7$ & $5.89 \pm 0.12$ & $0.55 \pm 0.14 \pm 0.12$ \\
$K^{-} K^{+} K^{-} \pi^{0}$ & 0 & 0 & 0 & $4.36 \pm 0.10$ & $<0.048(0.061)$ \\
\hline \hline
\end{tabular}

Table 1: Yields, backgrounds, efficiencies, and branching fraction measurements. The branching fraction for $\tau^{-} \rightarrow K^{-} \pi^{+} \pi^{-} \pi^{0} \nu_{\tau}$ excludes the $K^{0}$ and $\omega$ intermediate reasonances. The branching fraction for $\tau^{-} \rightarrow K^{-} K^{+} K^{-} \pi^{0} \nu_{\tau}$ corresponds to the $90 \%$ (95\%) C.L. upper limit. The second errors in the branching fraction measurements are systematic.

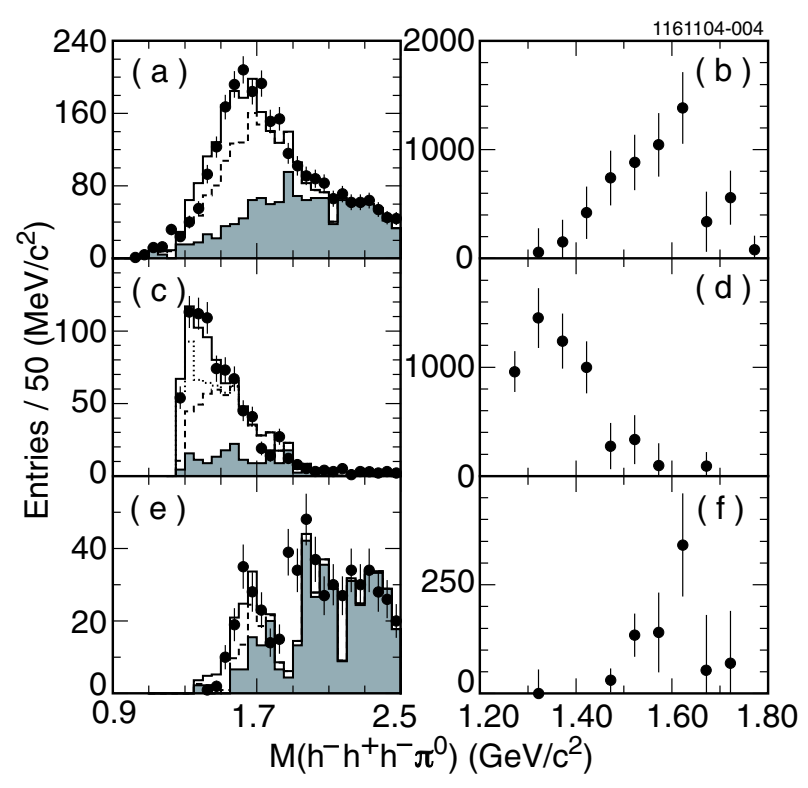

Figure 1: Four-hadron invariant mass spectra for $\tau^{-} \rightarrow K^{-} \pi^{+} \pi^{-} \pi^{0} \nu_{\tau}$ (ex. $\omega$ ) (a), $K^{-} \omega \nu_{\tau}$ (c), and $K^{-} K^{+} \pi^{-} \pi^{0} \nu_{\tau}$ (e) events. The histograms show the expectations, including $\tau$-migration Monte Carlo (dashed) and hadronic (shaded) background events. The dotted histogram in (c) shows the contribution of $K_{1}(1270)$. The distributions in (b, d, f) show the corresponding background- and efficiency-corrected spectra. Error bars are statistical only; (b, d, f) include errors in backgrounds and efficiencies.

tions for $\tau^{-} \rightarrow K^{-} \pi^{+} \pi^{-} \pi^{0} \nu_{\tau}$ (ex. $K^{0}$ ) and $K^{-} K^{+} \pi^{-} \pi^{0} \nu_{\tau}$. The decay $\tau^{-} \rightarrow K^{-} \omega \nu_{\tau}$ has also been observed for the first time. The branching fraction is nearly a factor of two smaller than Li's prediction [3], $\mathcal{B}\left(\tau^{-} \rightarrow K^{-} \omega \nu_{\tau}\right)=\mathcal{B}\left(\tau^{-} \rightarrow K^{-} \rho^{0} \nu_{\tau}\right)=7.5 \times 10^{-4}$, using a vector meson dominance model as well as $S U(3)_{f}$ relations between $\rho$ and $\omega$ mesons. Comparing the measurement with the previous measurement of $\tau^{-} \rightarrow K^{-} \rho^{0} \nu_{\tau}$ [10], the ratio of branching fractions, $\frac{\mathcal{B}\left(\tau^{-} \rightarrow K^{-} \omega \nu_{\tau}\right)}{\mathcal{B}\left(\tau^{-} \rightarrow K^{-} \rho^{0} \nu_{\tau}\right)}=0.26 \pm 0.11$, is likewise significantly lower than Li's prediction of 1 . The branching fractions, $\mathcal{B}\left(\tau^{-} \rightarrow K^{-} \pi^{+} \pi^{-} \pi^{0} \nu_{\tau}\right.$, ex. $\left.K^{0}, \omega\right)$ and $\mathcal{B}\left(\tau^{-} \rightarrow K^{-} \omega \nu_{\tau}\right) \times \mathcal{B}\left(\omega \rightarrow \pi^{+} \pi^{-} \pi^{0}\right)$, can be combined to yield $\mathcal{B}\left(\tau^{-} \rightarrow K^{-} \pi^{+} \pi^{-} \pi^{0} \nu_{\tau}\right.$, ex. $\left.K^{0}\right)=(7.4 \pm 0.8 \pm 1.1) \times 10^{-4}$, which is significantly more precise than previous measurements $[11,12]$. The measurement of $\mathcal{B}\left(\tau^{-} \rightarrow\right.$ 


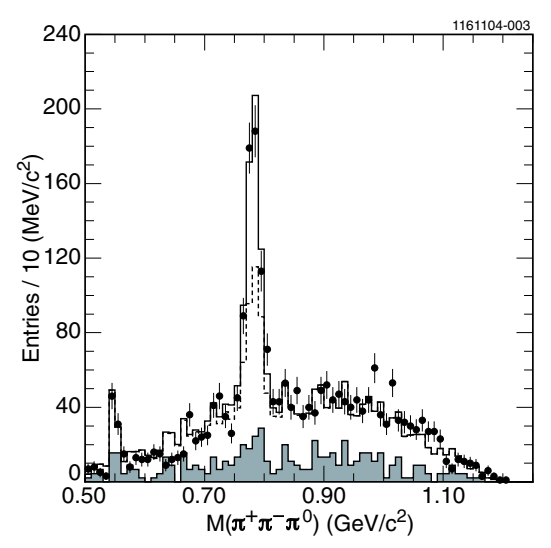

Figure 2: $\pi^{+} \pi^{-} \pi^{0}$ invariant mass spectrum in $\tau^{-} \rightarrow K^{-} \pi^{+} \pi^{-} \pi^{0} \nu_{\tau}$ events. The histogram shows the expectation using an equal mixture of $K_{1}(1400)$ and $K_{1}(1270)$, including $\tau$-migration Monte Carlo events (dashed) and hadronic background (shaded). Error bars are statistical only.

$\left.K^{-} K^{+} \pi^{-} \pi^{0} \nu_{\tau}\right)$ is almost an order of magnitude smaller than previous results [11, 12] which were based on samples with significantly larger $\tau$ migration backgrounds due to limited particle identification capability. The result for $\tau^{-} \rightarrow K^{-} K^{+} K^{-} \pi^{0} \nu_{\tau}$ corresponds to the first published upper limit on this decay. These new results, together with the first measurements of the invariant mass distributions, provide important ingredients which can be used to extract the Standard Model parameters, $m_{s}$ and $V_{u s}$.

\section{References}

[1] E. Gamiz et al., Phys. Rev. Lett. 94 (2005) 011803.

[2] Charge conjugate states are implied throughout this paper. For more details about this analysis, see K. Arms et al., Phys. Rev. Lett. 94 (2005) 241802.

[3] Bing An Li, Phys. Rev. $D 55$ (1997) 1436; The ratio $\frac{\mathcal{B}\left(\tau^{-} \rightarrow K^{-} \omega \nu_{\tau}\right)}{\mathcal{B}\left(\tau^{-} \rightarrow K^{-} \rho^{0} \nu_{\tau}\right)}$ should be 1 instead of $\frac{1}{3}$ in the paper according to private communication with the author.

[4] CLEO Collaboration, Y. Kubota et al., Nucl. Instrum. Meth. A 320 (1992) 66; D. Peterson et al., Nucl. Instrum. Meth. A 478 (2002) 142.

[5] T. Coan, Nucl. Instrum. Meth. A 379 (1996) 448; CLEO Collaboration, M. Artuso et al., Nucl. Instrum. Meth. A $\mathbf{5 0 2}$ (2003) 91.

[6] S. Jadach and Z. Was, Comput. Phys. Commun. 36 (1985) 191; 64, 267 (1991); S. Jadach, J. H. Kuhn, and Z. Was, ibid. 64 (1991) 275.

[7] R. Brun et al., CERN Report No. CERN-DD/EE/84-1, 1987 (unpublished).

[8] CLEO Collaboration, J.E. Bartelt et al., Phys. Rev. Lett. 76 (1996) 4119.

[9] ALEPH Collaboration, D. Buskulic et al., Z. Phys. C 74 (1997) 263.

[10] Particle Data Group, S. Eidelman et al., Phys. Lett. B 592 (2004) 1.

[11] CLEO Collaboration, S.J. Richichi et al., Phys. Rev. D 60 (1999) 112002.

[12] ALEPH Collaboration, R. Barate et al., Eur. Phys. J. C 1 (1998) 65. 